\title{
Effect of shRNA targeting mouse CD99L2 gene in a murine B cell lymphoma in vitro and in vivo
}

\author{
FANG LIU ${ }^{1,2^{*}}$, GONG ZHANG $^{1 *}$, FANRONG LIU $^{1}$, XINHUA ZHOU $^{1,3}$, XIAOYAN CHEN $^{1,2}$, \\ XIQUN HAN ${ }^{1,3}$, ZIQING WU ${ }^{1,3}$ and TONG ZHAO ${ }^{1,3}$ \\ ${ }^{1}$ Department of Pathology, School of Basic Medical Science, Southern Medical University, Guangzhou 510515; \\ ${ }^{2}$ Department of Physiology and Pathophysiology, Medical School, Foshan University, Foshan 528000; \\ ${ }^{3}$ Department of Pathology, Nanfang Hospital, Southern Medical University, Guangzhou 510515, P.R. China
}

Received November 7, 2012; Accepted December 11, 2012

DOI: $10.3892 /$ or.2013.2244

\begin{abstract}
Mouse CD99 antigen-like 2 ( $m$ CD99L2) has previously been confirmed to be expressed in murine B lymphoma (A20) cells by our group. The present study aimed to establish a mCD99L2-downregulated A20 cell line and to investigate the effect of shRNA targeting $m C D 99 L 2$ in A20 cells in vitro and in vivo. Four pLenti6/mCD99L2 expression vectors containing the mCD99L2 shRNA-expressing cassette were constructed, transfected into A20 cells and stable mCD99L2-downregulated A20 subclones, termed A20-mCD99L2- cells, were established and identified by quantitative PCR and western blot analysis. Light and transmission electron microscopy, MTT assay, flow cytometry and immunofluorenscence labeling were used to observe the morphological, biological and phenotypic characteristics in vitro. Some of the A20-mCD99L2 cells exhibited H/RS-cell like morphology, a decreased proliferative ability, a prolonged G2 phase and increased CD30 and CD15 expression. Upon injecting cells into nude or immunocompetent BALB/c mice, tumorigenesis, tumor growth, morphology and phenotypes in vivo were observed. A20-mCD99L2- cells induced tumors in nude and BALB/c mice, but with less potency in the latter compared with the controls. Similar morphological, biological and phenotypic characteristics were observed in the A20-mCD99L2 cell-induced tumors as those in vitro. Several cytokines including CD30T, IL-12p40/p70, IL-3, IFN- $\gamma$, CXCL16, MIP-1 $\alpha$ and CD40 were upregulated following mCD99L2 downregulation when detected using antibody
\end{abstract}

Correspondence to: Professor Tong Zhao or Dr Ziqing Wu, Department of Pathology, School of Basic Medical Science, Southern Medical University, 1838 North Guangzhou Dadao, Guangzhou 510515, P.R. China

E-mail: zhaotongketizu@126.com

E-mail: hailian@fimmu.com

*Contributed equally

Key words: lymphoma, A20 cell line, mouse CD99 antigen-like 2 gene, Hodgkin and Reed-Sternberg cell, nuclear factor- $\kappa \mathrm{B}$ arrays. The results from western blot analysis indicated that the regulation of mCD99L2 expression may involve the activated nuclear factor- $\kappa \mathrm{B}$ pathway in the murine B lymphoma cells. The present study provides data for further investigation into the mCD99L2 gene in tumor cells.

\section{Introduction}

Human CD99 is a $32 \mathrm{kDa}$ transmembrane glycoprotein encoded by a pseudoautosomal MIC2 gene located in Xp22.33-pter and Yp11-pter (1). It is expressed in several types of cells and is involved in several cellular events such as intercellular adhesion between lymphocytes and endothelial cells (2), migration of immune cells to inflammatory sites $(3,4)$ and attenuation of graft-versus-host disease (5). Certain studies have found that CD99 is expressed in tumors, including lymphoblastic lymphoma/leukaemia (6), Ewing's sarcoma/primitive neuroectodermal tumors (ES/PNET) (7), dermatofibrosarcoma protuberans, as well as giant cell fibroblastoma (8) and hepatoblastomas (9), while others found that CD99 is expressed in benign pancreatic endocrine neoplasm and gastric adenocarcinoma (10), but not in malignant counterparts.

Mouse CD99 antigen-like2 ( $m C D$ 99L2), a widely expressed antigen of unknown function with moderate sequence homology to CD99, was identified and cloned by Suh et al (11). mCD99L2 mRNA is widely distributed in neuronal cells, choroid plexus, sertoli cells and ovarian granulosa and thecal cells. It acts during leukocyte extravasation in mouse leukocytes and endothelial cells (12) to help neutrophils penetrate the endothelial basement membrane (13). Amino acid sequence alignment showed five putative functional regions highly conserved between mCD99L2 and human CD99, implying they may have similar functions. However, to date, the majority of studies on mCD99L2 have focused mainly on its roles in inflammation. Its effects on tumor cells have yet to be reported.

A20 is a murine cell line derived from a spontaneously arising tumor in an aged BALB/c mouse (14). It pathologically mimics the characteristics of human diffuse large $\mathrm{B}$ cell lymphoma (15). An A20-related animal model may be used in studies on the association between tumors and hosts (16). We successfully established a disseminated A20 animal model and described its immuno-characteristics (17). We also 
observed that mCD99L2 was expressed in A20 cells (18). To investigate the role of mCD99L2 in A20 cells, four pLenti6/ mCD99L2 expression vectors containing the mCD99L2 shRNA-expressing cassette were constructed in the present study, transfected into A20 cells using DMRIE-C2, and the stable mCD99L2-downregulated A20 cell line, termed A20-mCD99L2 cells, was established and identified using quantitative PCR and western blot analysis. The effect of shRNA targeting mCD99L2 during continuous culturing was observed. The morphological, biological and phenotypic characteristics of the A20-mCD99L2- cells were extensively investigated in vitro and in vivo, to provide data for additional functional studies of mCD99L2 in associated tumors.

Using CD99-deficient IM9 and BJAB B cell lines, investigators have confirmed that the downregulation of CD99 is a primary requirement for generating 'Hodgkin and Reed-Sternberg' (H/RS) cells $(19,20)$. A previous study of ours suggested that the CD99-upregulated H/RS cell line (L428) lost its nature as H/RS cells (21). In this study, we investigated whether mCD99L2 downregulation can induce cells with an H/RS morphology and phenotypes in murine B lymphoma cells, to determine whether there is a functional similarity between human CD99 and murine CD99 genes.

\section{Materials and methods}

Cell lines and morphology observation. The BALB/c-derived mouse B lymphoma A20 cell line was kindly provided by Professor Chan of the Nebraska Medical Center, Omaha, NE, USA. The subclones of A20 cells transfected with shRNAs targeting mCD99L2 or negative control vectors were constructed and cultured in RPMI-1640 medium supplemented with 10\% heat-inactivated fetal bovine serum (FBS) (Gibco, Grand Island, NY, USA).

Morphological observation of live cells was conducted with an inverted microscope. Transmission electron microscopy (TEM) was performed according to the manufacturer's instructions. Hematoxylin and eosin (H\&E) staining was applied on the fixed cells previously dripped onto slides and preserved under $-80^{\circ} \mathrm{C}$.

Preparation of lentiviral vectors and RNA interference (RNAi). Four different sequences targeting the mCD99L2 gene were selected by BLOCK-iT ${ }^{\mathrm{TM}}$ RNAi Designer (Invitrogen, Carlsbad, CA, USA). The preparation of lentiviral vectors expressing mCD99L2 short hairpin RNA (shRNA) was performed using the BLOCK-iT Lentiviral RNAi Expression System (catalog no. K4944-00; Invitrogen). Four pLenti6/ mCD99L2 expression vectors containing the mCD99L2 shRNA-expressing cassette were constructed. The lentiviral vectors containing the human Lamin A/C shRNA-expressing cassette (sequence 5'-CTGGACTTCCAGAAGAACA-3') were used as the positive control and the pLenti6/U6 mock vector was used as the negative control. A20 cells were transfected with specific or negative control lentiviral vectors using DMRIE-C2 at suitable ratios and selected for stable integrants by culturing in complete medium containing blasticidin (Invitrogen). Several single blasticidin-resistant colonies were isolated using the soft agar clone formation protocol, expanded into sub-cell lines by 96 -well plate limiting dilution assay.
RNA isolation and quantitative PCR. Total RNA was extracted using the Takara RNAiso plus kit and cDNA was prepared from $2 \mu \mathrm{g}$ total RNA by PrimeScript Reverse Transcriptase (Takara Bio Co., Ltd., Shiga, Japan). Real-time PCR was performed on a 7500/7500 Fast Real-Time PCR System (Applied Biosystem, Foster City, CA, USA) using a SYBR-Green Premix Ex Taq ${ }^{\mathrm{TM}}$ kit (Takara), following the manufacturer's instructions under the conditions of $95^{\circ} \mathrm{C}$ for $30 \mathrm{sec}$, followed by 40 cycles of $95^{\circ} \mathrm{C}$ for $5 \mathrm{sec}$ and $58^{\circ} \mathrm{C}$ for $34 \mathrm{sec}$. PCR primers were purchased from Invitrogen and were as follows: mCD99L2, forward, 5'-GCCCAGCAACAAGCAAAGCACAT-3' and reverse, 5'-CCCAACCACCCTAGTTCCTCCG-3'; GAPDH, forward, 5'-ACAGTCAGCCGCATCTTCTT-3' and reverse, 5'-GACAA GCTTCCCGTTCTCAG-3'. The results were analyzed using the software installed in the 7500/7500 Fast Real-Time PCR System (Applied Biosystems) and the relative expression ratio was determined using the formula $2^{-\Delta \Delta \mathrm{Ct}}$.

Cell proliferation. Cell proliferation was analyzed using the MTT assay (Sigma, St. Louis, MO, USA). Briefly, $1 \times 10^{3}$ cells were seeded into each well of a 96-well plate with quadruplicate repeats for each condition. After $24 \mathrm{~h}$ of incubation, cells were mixed with MTT reagent and incubated for $4 \mathrm{~h}$. The formazan crystals formed by viable cells were then solubilized in dimethyl sulfoxide (DMSO) and measured at $490 \mathrm{~nm}$. Each experiment was performed in triplicate.

Flow cytometry. The cultured cells were harvested at the exponential growth phase and prepared as single cell suspensions. Cells $\left(1 \times 10^{6}\right)$ were fixed in $70 \%$ ethanol in phosphate-buffered saline(PBS) on ice, pelleted,incubated with RNase A $(0.1 \mu \mathrm{g} / \mathrm{ml})$ for $30 \mathrm{~min}$ at $37^{\circ} \mathrm{C}$ and stained with propidium iodide (PI) $(40 \mu \mathrm{g} / \mathrm{ml})$ for cell cycle analysis.

Cells were stained with panels containing fluorescein isothiocyanate (FITC)-conjugated anti-mouse antibodies against CD19 and CD20, R-Phycoerythrin (PE)-conjugated anti-mouse antibodies against CD30 and CD15, and control FITC- or PE-conjugated mouse IgG1 (BD Pharmingen, San Diego, CA, USA), as indicated. CD antigen expressions were analyzed on a FACSCalibur machine (ELITE; BeckmanCoulter, Fullerton, CA, USA).

Immunofluorescence. Cells $\left(2.0 \times 10^{5} / \mathrm{ml}\right)$ were inoculated into each well of 6-well plates (Costar, Corning, NY, USA) and cultured in complete medium for $48 \mathrm{~h}$ followed by in serumfree medium for another $24 \mathrm{~h}$. After deposition, fixation and permeabilization, the cells were labeled with rabbit anti-mouse CD30 mAb (2 $\mu \mathrm{g} / \mathrm{ml}$; Abcam) followed with PE-conjugated goat anti-rabbit IgG (15 $\mu \mathrm{g} / \mathrm{ml}, \mathrm{ZF}-0311$; ZSGB-BIO, Beijing, China). Negative controls were performed by replacing the primary antibodies with PBS. The cells were observed under a fluorescence microscope (Nikon, Tokyo, Japan).

Mouse cytokine antibody arrays. RayBio ${ }^{\circledR}$ Mouse Cytokine Antibody Arrays (RayBiotech, Inc., Norcross, GA, USA) were used to investigate the expression of 62 cytokines in the different cell groups, according to the manufacturer's instructions. In brief, proteins were extracted, quantified and transferred onto membranes. The membranes were then sealed and incubated with antibodies against cytokines. The diffe- 
Table I. Sequences of shRNA targeting mCD99L2.

\begin{tabular}{lll}
\hline No. & \multicolumn{1}{c}{ Sequences } \\
\hline 1 & Top strand & 5'-CACCGCCTTGTCCAGAGAGGATATCGAAATATCCTCTCTGGACAAGG-3' \\
& Bottom strand & 5'-AAAACCTTGTCCAGAGAGGATATTTCGATATCCTCTCTGGACAAGGC-3' \\
2 & Top strand & 5'-CACCGCCACTACTACAACTAGAACGAATTCTAGTTGTAGTAGTGGC-3' \\
& Bottom strand & 5'-AAAAGCCACTACTACAACTAGAATTCGTTCTAGTTGTAGTAGTGGC-3' \\
3 & Top strand & 5'-CACCGGAAGATGCCTTGGATGATCGAAATCATCCAAGGCATCTTCC-3' \\
& Bottom strand & 5'-AAAAGGAAGATGCCTTGGATGATTTCGATCATCCAAGGCATCTTCC-3' \\
4 & Top strand & 5'-CACCGCCTTGCTATGGCCCTGATTCGAAAATCAGGGCCATAGCAAGG-3' \\
& Bottom strand & 5'-AAAACCTTGCTATGGCCCTGATTTTCGAATCAGGGCCATAGCAAGGC-3'
\end{tabular}

Bold letters indicate sense; letters in italics indicate loop; underlined letters indicate antisense. mCD99L2, mouse CD99 antigen-like 2.

rences in cytokine expression were visualized, photographed and analyzed.

Western blot analysis. Cell lysates were prepared, and equal amounts of protein $(50 \mu \mathrm{g})$ were separated on $10 \%$ sodium dodecyl sulfate-polyacrylamide gel electrophoresis (SDS-PAGE), and transferred onto polyvinylidene difluoride (PVDF) membranes (Bio-Rad Laboratories, Hercules, CA, USA). Membranes were incubated with $5 \%$ skim milk in TBS- $0.1 \%$ Tween-20 for $2 \mathrm{~h}$ to block the residual binding sites followed by immunoblotting overnight at $4{ }^{\circ} \mathrm{C}$ with appropriately diluted rabbit anti-human $\mathrm{p}-\mathrm{I} \kappa \mathrm{B} \alpha$ antibody (1:500; Bioworld Technology, Inc., St. Louis Park, MN, USA), rabbit mCD99L2 antibody (Abcam, Cambridge, MA, USA) and rabbit $\beta$-actin antibody (ZhongShan Golden Bridge Biotechnology, Bejing, China). Specific binding was revealed by mouse HRP-conjugated anti-rabbit IgG (Santa Cruz Biotechnology, Inc., Santa Cruz, CA, USA) and an enhanced chemiluminescence system (ECL-Plus; Amersham Biosciences Inc., Piscataway, NJ, USA).

Animals and in vivo tests. Twelve nude mice and $84 \mathrm{BALB} / \mathrm{c}$ mice (six to eight-week-old female/male) were purchased from the Central Laboratory of Animal Science of the Southern Medical University (Guangzhou, China) and randomized into an A20-mCD99L2- group and an A20-empty group of nude and BALB/c mice, respectively (Table I). Tumor cells $\left(2 \times 10^{6}\right.$ to $2 \times 10^{7}$ ) in $0.1-0.4 \mathrm{ml}$ growth medium were injected into the mice using various methods. Tumor growth was observed by calculating the tumor volume. Mice were sacrificed when exhibiting external signs of suffering (such as reduced mobility and altered behavior). The procedures were conducted under sterile conditions. The animal protocol for this experiment was approved by the Animal Care and Use Committee of the Southern Medical University.

Immunohistochemistry. Tissue specimens were collected from tumors in the mice and fixed in formalin and embedded in paraffin. The tissues were then cut into $2-\mu \mathrm{m}$ sections and dried on capillary-gap glass slides. Immunochemistry of the paraffin sections was carried out using the ChemMate ${ }^{\mathrm{TM}}$ EnVision $^{\mathrm{TM}}$ Detection kit (Dako, Carpinteria, CA, USA).
The sections were dewaxed, dehydrated, subjected to antigen retrieval and blocked for endogenous peroxidase activity. The sections were then immunostained with CD3 antibody (brown color) with DAB substrate (Dako) and then counterstained using hematoxylin.

Statistical analysis. The SPSS 13.0 software was used for statistical analysis. The results are expressed as the means \pm standard deviation (SD). Where indicated, differences were compared using the Student's t-test. Assay differences between in vitro cell growth and in vivo tumor growth were examined for statistical significance using analysis of variance (ANOVA) for factorial design. Proliferation assay and FACS results of antigen expression or lymphocyte percentages were examined using one-way ANOVA. Statistical analysis of tumor growth was carried out using Dunnett's multiple comparison tests. $\mathrm{P}<0.05$ was considered to indicate a statistically significant difference.

\section{Results}

Establishment of A20 subclones using shRNA targeting $m C D 99 L 2$ gene. Four primers were designed based on the RNAi technique, shRNA was constructed and sequencing was confirmed followed by transient transfection into A20 cells (Table II). Interference efficacy was examined using real-time RT-PCR until targeting sequences were screened. No. 2 lentiviral vector was most effective at blocking $m C D 99 L 2$ expression (Table II, no. 2 shRNA). Subsequently, the pLenti6/mCD99L2 (no. 2) and pLenti6/U6 mock vector were transfected into the A20 cells and blasticidin-resistant single clones were selected, achieving permanent transfection to gain stable integrants. The transfection rate was $57 \%$ at 16 days assayed by PI staining flow cytometry. For the sake of convenience, clones transfected using pLenti6/mCD99L2 or pLenti6/U6 mock vector were termed A20-mCD99L2- cells and A20-empty cells, respectively. Stable integrants of low mCD99L2 expression of the A20 cells were achieved (A20-mCD99L2- cells) and confirmed in various clones. $m C D 99 L 2$ expression was significantly lower in the A20-mCD99L2- cell group compared with the A20 and A20-empty groups; it decreased by $50 \%$, as indicated by realtime PCR and western blot analysis (Fig. 1). 
Table II. Assignment of animals groups.

\begin{tabular}{lllll}
\hline Group & \multicolumn{1}{c}{ Animal (no.) } & \multicolumn{1}{c}{ Methods } & $\begin{array}{c}\text { Cells inoculated } \\
\text { (/mice) }\end{array}$ & Inoculation site \\
\hline A & Nude mouse (6) & Subcutaneous inoculation & $2 \times 10^{7} / 0.2 \mathrm{ml}$ & Axillary fossa \\
B1 & BALB/c mouse (7) & Subcutaneous inoculation & $2 \times 10^{6} / 0.1 \mathrm{ml}$ & Left axillary fossa \\
B2 & BALB/c mouse (7) & Subcutaneous inoculation & $2 \times 10^{7} / 0.1 \mathrm{ml}$ & Left axillary fossa \\
C1 & BALB/c mouse (7) & Subcutaneous transplantation & Tumor tissue & Left axillary fossa \\
C2 & BALB/c mouse (7) & Subcutaneous transplantation & Tumor tissue & Right axillary fossa \\
D1 & BALB/c mouse (7) & Subcutaneous transplantation & $5 \times 10^{6} / 0.2 \mathrm{ml}$ & Caudal vein \\
D2 & BALB/c mouse (7) & Subcutaneous transplantation & $2 \times 10^{7} / 0.4 \mathrm{ml}$ & Caudal vein \\
\hline
\end{tabular}

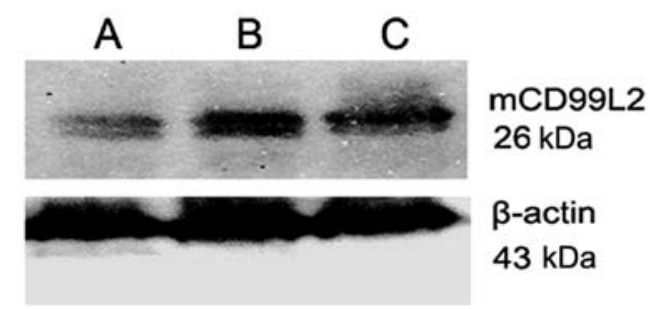

Figure 1. Western blot analysis of mCD99L2 protein. Western blot analysis of mCD99L2 protein $(26 \mathrm{kDa})$ and the control, $\beta$-actin $(43 \mathrm{kDa})$ in (A) A20-mCD99L2, (B) A20 and (C) A20-empty cells.

Morphological changes by downregulating mCD99L2 expression in vitro. Morphology was extremely significant in the present study. Some cells in the A20-mCD99L2- group demonstrated morphological changes including larger volume, abundant cytoplasm, marked pleomorphism, large and deeply stained nucleus, while some were binucleated or polynucleated (Fig. 2A). Even when cells were continuously cultured to the 20th passages (Fig. 2B), these changes in various clones of the A20-mCD99L2 group remained. TEM observation indicated that controls were naïve cells with less cytoplasm and organelles and relatively larger nucleus, while the giant cells in the A20-mCD99L2- group were much larger, with abundant cytoplasm and organelles, particularly mitochondria and endoplasmic reticulum (Fig. 2C). The unique morphology of binucleated or polynucleated appearance with larger nucleus and nucleoli exhibited by some A20-mCD99L2 cells partly mimic that of human $\mathrm{H} / \mathrm{RS}$ cells.

Biological characteristics of A20- $m$ CD 99L2 cells. The growth of cultured A20-mCD99L2 cells was investigated using the MTT method. Slower kinetics of cell proliferation compared with the controls were observed in the A20-mCD99L2 group (Table III) $(\mathrm{P}<0.05)$, which indicated that mCD99L2 downregulation induces a weaker proliferative ability.

To evaluate the cell cycle distribution, the DNA contents of asynchronous cultures of various cell groups were measured. The $\mathrm{S}$ phase of each group showed no significant difference $(\mathrm{P}>0.05, \mathrm{n}=4)$, while the $\mathrm{G} 2$ phase was significantly prolonged in the A20-mCD99L2 cells compared to the A20 and A20-empty cells (Table IV) $(\mathrm{P}<0.05)$, which indicated that A20-mCD99L2 cells may be defective in cytokinesis.
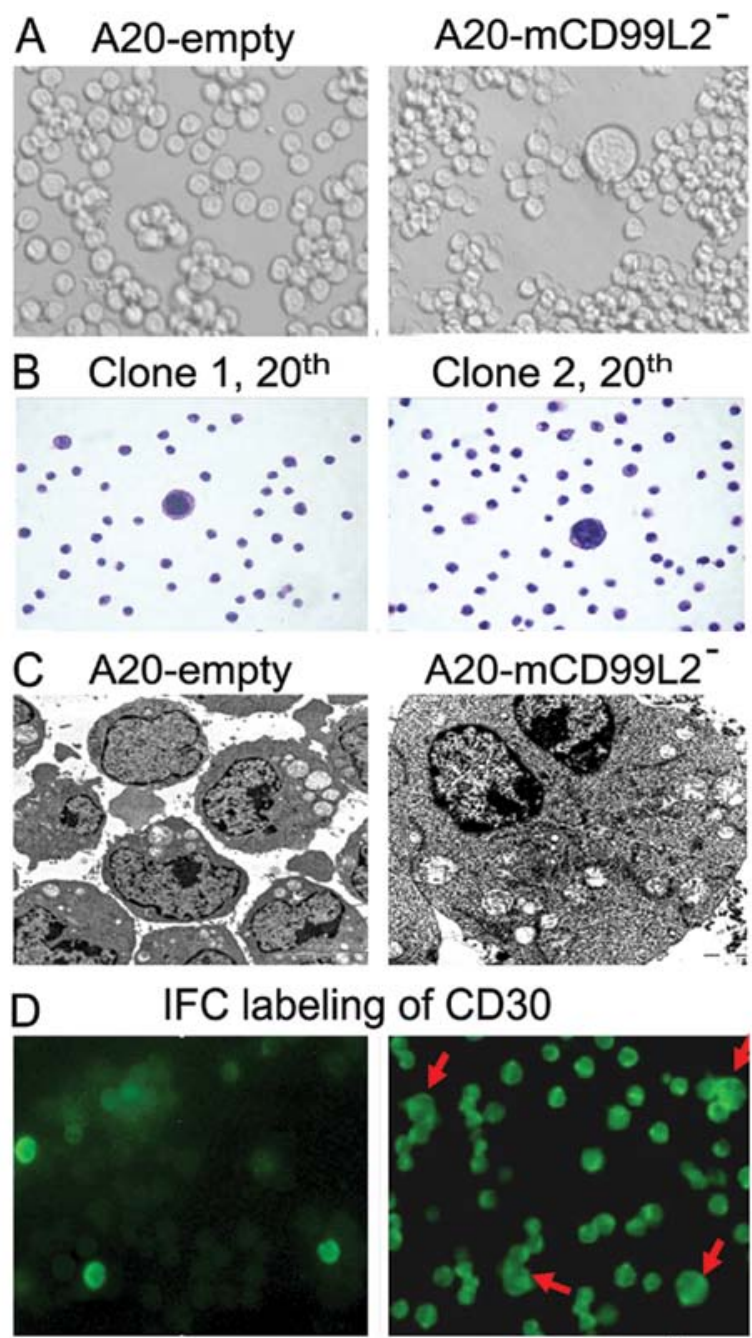

A20-empty cell
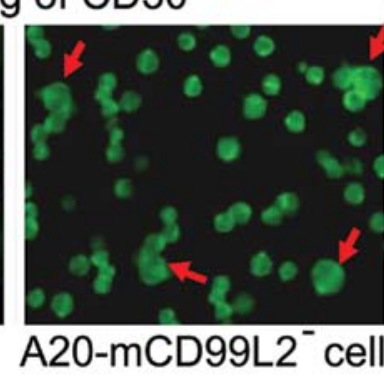

Figure 2. Morphological changes and immunofluroscence (IFC) labeling of CD30. (A) The morphological changes under an inverted microscope (x400). (B) H\&E staining of different clones of A20-mCD99L2 cells during continuous passage of culture (x200). (C) Observation by transmission electron microscopy (x8,000). (D) IFC labeling of CD30.

Immunophenotypes of A20-mCD99L2 cells in vitro. As $\mathrm{H} / \mathrm{RS}$ cells are characterized by a high expression of CD15 and CD30 (22) and previous findings have demonstrated that upreg- 


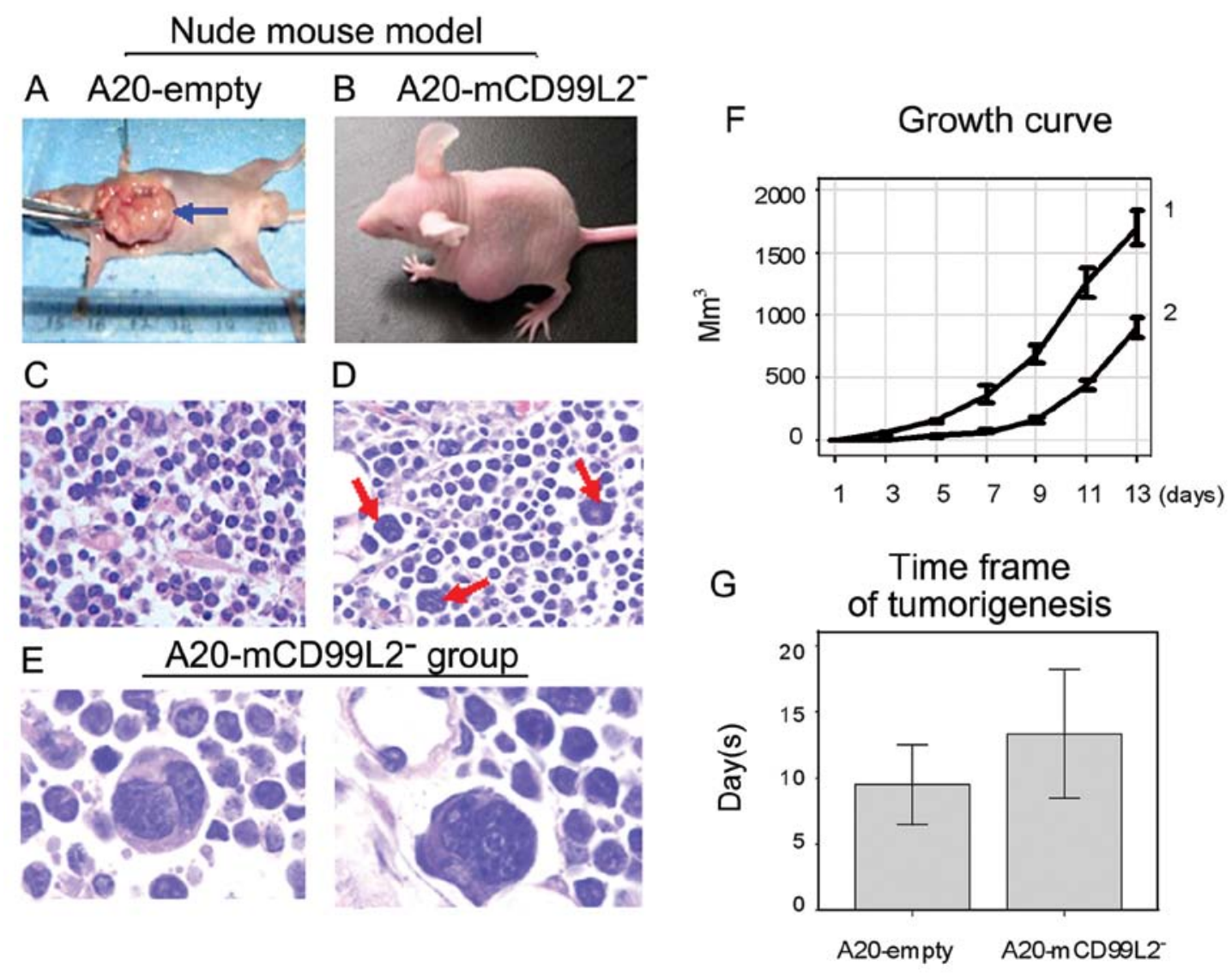

Figure 3. Histology and biology of tumors in nude mice. (A and B) Gross anatomy of nude mouse and (C-E) hematoxylin and eosin (H\&E) staining of tumor tissues following subcutaneous inoculating of A20-empty and A20-mCD99L2- cells (C and D) x400, (E) x1,000. (F) Growth curve of tumors of (line 1, A20empty; line 2, A20-mCD99L2- cells). Vertical axis, tumor volume $\left(\mathrm{mm}^{3}\right)$. (G) Time frame of tumorigenesis of various groups' vertical axis, day(s).

Table III. Proliferation of various cell groups in vitro by MTT $(\mathrm{n}=6)$.

\begin{tabular}{lccc}
\hline Day & A20 & A20-empty & A20-mCD99L2- \\
\hline 1 & $0.43 \pm 0.02$ & $0.43 \pm 0.02$ & $0.43 \pm 0.02$ \\
2 & $0.53 \pm 0.02$ & $0.53 \pm 0.03$ & $0.47 \pm 0.02^{\mathrm{a}, \mathrm{b}}$ \\
3 & $0.65 \pm 0.02$ & $0.64 \pm 0.03$ & $0.55 \pm 0.04^{\mathrm{a}, \mathrm{b}}$ \\
4 & $0.84 \pm 0.02$ & $0.83 \pm 0.03$ & $0.65 \pm 0.03^{\mathrm{a}, \mathrm{b}}$ \\
5 & $0.95 \pm 0.02$ & $0.93 \pm 0.03$ & $0.73 \pm 0.05^{\mathrm{a}, \mathrm{b}}$ \\
6 & $0.94 \pm 0.03$ & $0.92 \pm 0.01$ & $0.71 \pm 0.03^{\mathrm{a}, \mathrm{b}}$ \\
7 & $0.92 \pm 0.02$ & $0.92 \pm 0.02$ & $0.70 \pm 0.02^{\mathrm{a}, \mathrm{b}}$ \\
\hline
\end{tabular}

ap-value of time, $\mathrm{P}<0.05$; ${ }^{\mathrm{P}} \mathrm{P}$-value between groups, $\mathrm{P}<0.05$. mCD99L2, mouse CD99 antigen-like 2.

Table IV. Cell cycle analysis of various cell groups $(\mathrm{n}=4)$.

\begin{tabular}{lccc}
\hline Cell groups & G1 $(\%)$ & $\mathrm{S}(\%)$ & $\mathrm{G} 2(\%)$ \\
\hline A20 & $42.60 \pm 4.23$ & $54.05 \pm 4.86$ & $3.33 \pm 1.31^{\mathrm{a}}$ \\
A20-empty & $41.63 \pm 3.93$ & $55.03 \pm 4.10$ & $3.32 \pm 1.30^{\mathrm{b}}$ \\
A20-mCD99L2 & $35.90 \pm 3.13$ & $53.55 \pm 5.50$ & $10.58 \pm 4.97^{\mathrm{a}, \mathrm{b}}$ \\
\hline
\end{tabular}

${ }^{\mathrm{a}} \mathrm{P}<0.05$ and ${ }^{\mathrm{b}} \mathrm{P}<0.05$. mCD99L2, mouse CD99 antigen-like 2. ulated CD99 markedly downregulates the expression of CD30 and CD15 (19-21), flow cytometry was applied to examine the changes in $\mathrm{CD}$ antigen expression by downregulating mCD99L2. The results indicated that when compared with the controls, A20-mCD99L2- cells exhibited significantly higher CD30 and CD15 levels (Table V) $(\mathrm{P}<0.01, \mathrm{n}=3)$ and moderately decreased CD19 and CD20 levels $(\mathrm{P}<0.05, \mathrm{n}=3)$. The enhanced expression of $\mathrm{CD} 30$ was also confirmed by immunofluoresence (IFC) labeling using mouse CD30 antibodies and the giant cells as well as some transformed A20-mCD99L2 cells were CD $30^{+}$(Fig. 2D).

Histology, biology and phenotypes of tumors in nude mice. Subcutaneous tumor models in nude mice were successfully established (Table VI, Fig. 3A-E). Tumor growth of the A20-mCD99L2- group was much slower than the A20-empty group (Fig. 3F). The time frame of tumorigenesis in the A20-mCD99L2- group was much longer compared with the control group (Fig. 3G), which indicated that the proliferative ability of the A20-mCD99L2 cells was weaker due to the downregulation of mCD99L2.

Histologically, the A20-empty tumors were characterized by a diffuse homogeneous infiltrate consisting of large and cohesive tumor cells with moderate cytoplasm and pleomorphic nuclei (Fig. 3C), while the A20-mCD99L2- tumor cells showed marked pleomorphism, a diffuse distribution pattern, and some had two or more large and deeply stained nuclei (Fig. 3D and E). 
Table V. Analysis of the antigen expression of each group using FACS ( $n=3)$.

\begin{tabular}{lcccc}
\hline Group & CD19 & CD20 & CD30 & CD15 \\
\hline A20 & $91.57 \pm 2.11$ & $41.33 \pm 2.25$ & $41.70 \pm 2.60$ & $51.2 \pm 2.60$ \\
A20-empty & $90.90 \pm 4.41$ & $40.80 \pm 3.90$ & $41.87 \pm 2.06$ & $49.9 \pm 2.92$ \\
A20-mCD99L2- & $75.70 \pm 3.2^{\mathrm{a}}$ & $13.10 \pm 5.16^{\mathrm{a}}$ & $76.0 \pm 2.44^{\mathrm{a}}$ & $71.6 \pm 3.60^{\mathrm{a}}$ \\
\hline
\end{tabular}

${ }^{\text {aP }}<0.05$. mCD99L2, mouse CD99 antigen-like 2 .

Table VI. Tumorigenesis in each mouse group inoculated with various tumor cells.

\begin{tabular}{|c|c|c|c|c|c|}
\hline \multirow[b]{2}{*}{ Group } & \multirow[b]{2}{*}{$\mathrm{N}$ (total) } & \multicolumn{2}{|c|}{ Percentage of tumorigenesis $(\%)(\mathrm{n})$} & \multicolumn{2}{|c|}{ Time frame of tumorigenesis (days) } \\
\hline & & A20-empty & A20-mCD99L2- & A20-empty & A20-mCD99L2- \\
\hline A & 12 & $100(6 / 6)$ & $100(6 / 6)$ & $9.5 \pm 2.9$ & $13.33 \pm 4.63$ \\
\hline B1 & 14 & $0(0 / 7)$ & $0(0 / 7)$ & - & - \\
\hline B2 & 14 & $100(7 / 7)$ & $14.3(1 / 7)$ & $15.29 \pm 3.2$ & 10 \\
\hline $\mathrm{C} 1$ & 14 & $100(7 / 7)$ & $14.3(1 / 7)$ & $7.0 \pm 0.82$ & 6 \\
\hline $\mathrm{C} 2$ & 14 & $100(7 / 7)$ & $0(0 / 7)$ & $6.29 \pm 0.49$ & - \\
\hline D1 & 14 & $71.4(5 / 7)$ & $0(0 / 7)$ & $76.8 \pm 12.0$ & $>3$ months \\
\hline D2 & 14 & $100(7 / 7)$ & $0(0 / 7)$ & $26.1 \pm 7.9$ & $>3$ months \\
\hline
\end{tabular}

mCD99L2, mouse CD99 antigen-like 2.

The immunophenotypes of the primary tumor cells dissociated from the xenotransplanted tumors were examined using flow cytometry. CD30 expression was significantly upregulated, whereas CD19 expression was lower in the A20-mCD99L2compared with the A20-empty group.

Effect of downregulation of $m C D 99 L 2$ in BALB/c mice. Inoculating BALB/c mice with A20-mCD99L2 cells and A20-empty cells was conducted using various methods (Table II, Fig. 4A and B). It was difficult for the A20-mCD99L2cells to form tumors in $\mathrm{BALB} / \mathrm{c}$ mice irrespective of the methods applied (Table VI), indicating that the proliferative ability of the A20 cells with decreased mCD99L2 expression was significantly impaired in the immunocompetent BALB/c mice.

The histological characteristics of the A20-empty cell-induced tumor tissues demonstrated uniform B lymphoid cells, which were most consistent with those in human diffuse large B cell lymphoma (DLBCL) (Fig. 4C). Cells in A20-mCD99L2- tumor tissues exhibited marked pleomorphism and large and deeply stained nuclei, some were binucleated or polynucleated (Fig. 4D and E, red arrow). In addition, some lymphocytes were observed in the tumor tissues (Fig. 4D, black arrow).

CD30 was positive in A20-mCD99L2- cell-induced tumors as observed using IFC labeling of mouse CD30, particularly in the giant cells (Fig. 4F). As in Hodgkin's lymphoma, H/RS cells were accompanied by a number of background cells, including $\mathrm{T}$ lymphocytes. Immunohistochemistry was applied to detect the expression of $\mathrm{CD}^{+} \mathrm{T}$ lymphocytes in tumor tissues. The results indicated that more $\mathrm{CD}^{+}$lympho- cytes infiltrated into the A20-mCD99L2 - cell-induced tumor tissues compared with the control group (Fig. 4G), which suggested that various immune reactions were induced in vivo.

Differential cytokine profile of cells and tissues. The weak proliferative ability and pathological features exhibited in the A20-mCD99L2- groups, which partly mimicked those of Hodgkin's lymphoma, encouraged us to investigate the differential expression of cytokines/chemokines (Fig. 5A and B) induced by the downregulation of mCD99L2, as several cytokines are involved in the cross-talk between H/RS cells and the background inflammatory environment. Cytokine protein arrays showed that several cytokines including CD30T, IL-12p40/p70, IL-3, IFN- $\gamma$, CXCL16, MIP-1 $\alpha$ and CD40, were upregulated ( $\geq 1.5$-fold) in the A20-mCD99L2 cells and no cytokine was downregulated in excess of 1.5-fold (Table VII). The changes in morphology, biology and phenotypes may be directly or indirectly associated with these differentially expressed cytokines/chemokines.

mCD99L2 downregulation correlates with the activated nuclear factor $-\kappa B(N F-\kappa B)$ pathway. As regards the mecharnism involved, the NF- $\kappa \mathrm{B}$ pathway plays a crucial role in the pathology of Hodgkin's lymphoma and is a significant factor affecting cytokines/chemokines. $\mathrm{NF}-\kappa \mathrm{B}$ is retained in the cytoplasm of inactivated cells through the interaction with members of the I $\mathrm{B}$ inhibitor family, including $\mathrm{I} \kappa \mathrm{B} \alpha$. The phosphorylation and subsequent degradation of I $\mathrm{B}$ lead to the release of $\mathrm{NF}-\kappa \mathrm{B}$, allowing it to translocate to the nucleus and activate transcription. The level of $\mathrm{p}-\mathrm{I} \kappa \mathrm{B} \alpha$, which could 


\section{BALB/ c mouse model}
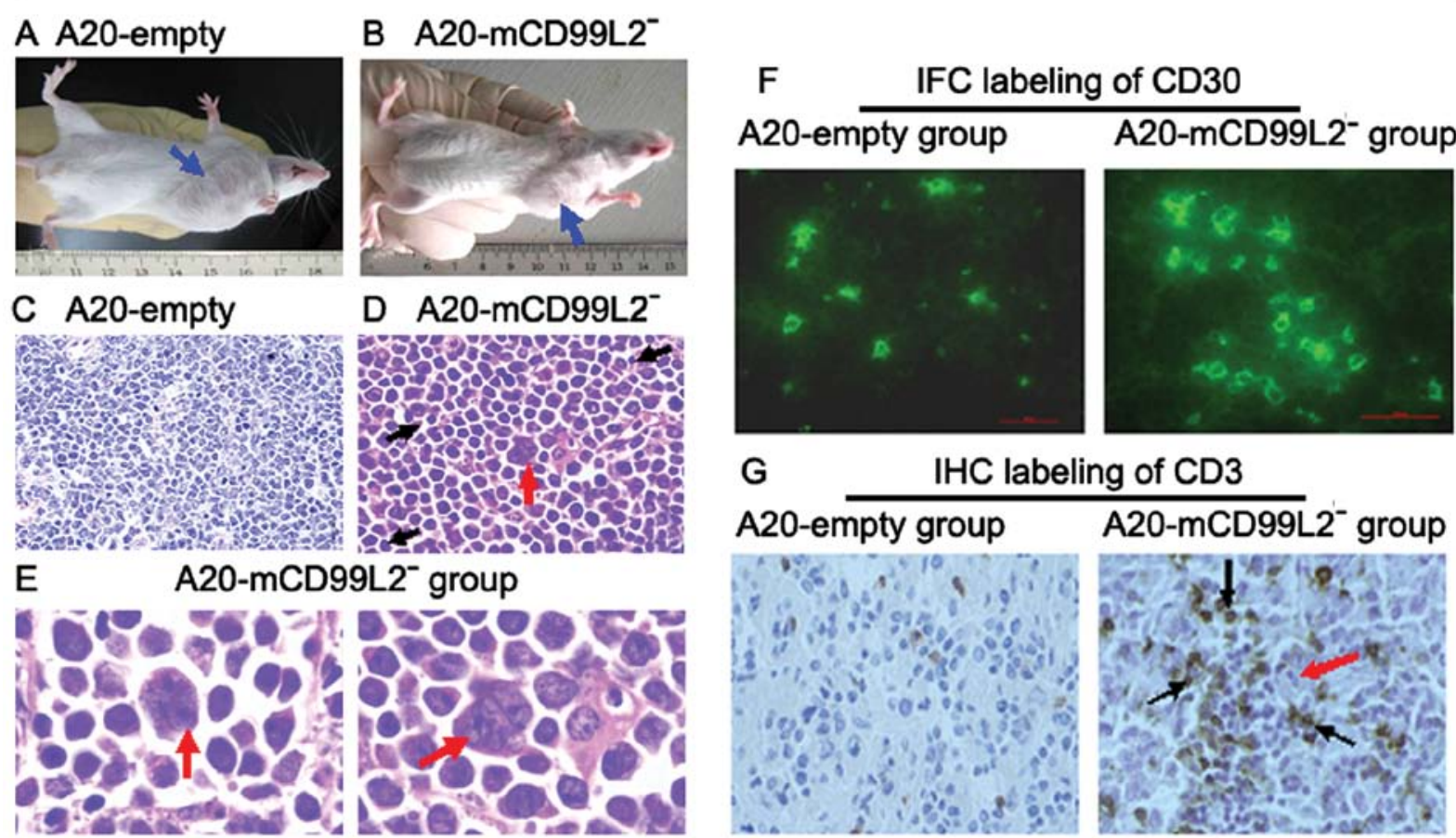

G
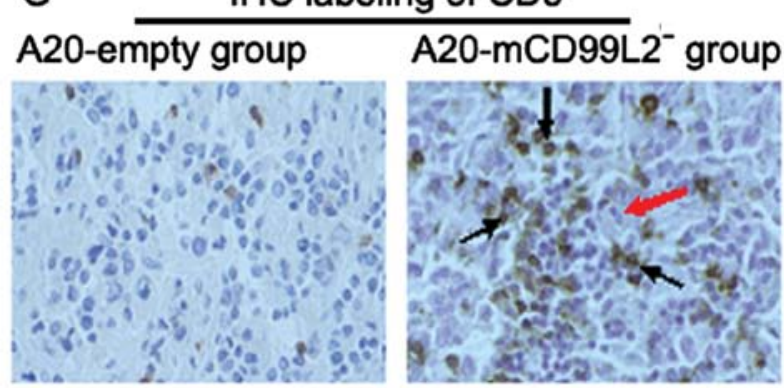

Figure 4. Histology and immunophenotypes of tumor tissues in BALB/c mice. Representative tumors (A and B, blue arrow) and hematoxylin and eosin (H\&E) staining (C-E) in BALB/c mouse subcutaneously injected with A20-empty and A20-mCD99L2- cells (C-D) x400, (E) x1,000, respectively. (Red arrow indicates binucleated or polynucleated cells in tumor tissues of the A20-mCD99L2- group; black arrow shows some infiltrated lymphocytes). (F) Immunofluorescence (IFC) labeling of mouse CD30 in tumor tissues. (G) Immunohistochemical (IHC) staining of CD3 in tumor tissues.
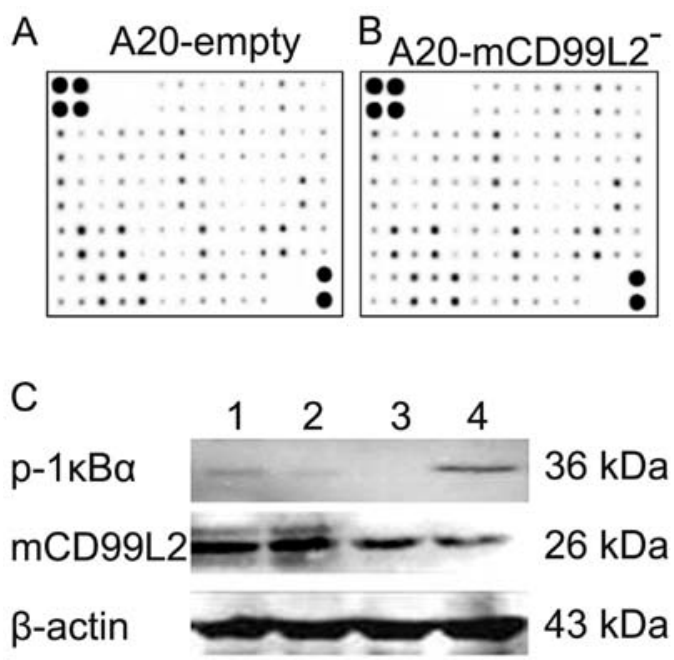

Figure 5. Cytokine expression investigated using antibody arrays and expression of $\mathrm{p}-\mathrm{I} \kappa \mathrm{B} \alpha$ analyzed using western blot analysis. Sixty-two types of cytokine/chemokine profiles of cultured (A) A20-empty cells and (B) A20-mCD99L2 cells. (C) Expression of $\mathrm{p}-\mathrm{I} \kappa \mathrm{B} \alpha(36 \mathrm{kDa})$ and mCD99L2 $(26 \mathrm{kDa})$ protein analyzed by western blot analysis in A20 cells (lane 1), A20-empty cells (lane 2), A20-mCD99L2 cells treated with inhibitor (BAY) of NF- $\mathrm{KB}$ pathway (lane 3) and A20-mCD99L2 cells (lane 4), the control $\beta$-actin $(43 \mathrm{kDa})$.

represent the activation of $\mathrm{NF}-\kappa \mathrm{B}$ pathway, was examined in various cell groups using western blot analysis (Fig. 5C).

The results indicated that the expression of the mCD99L2 protein in the A20-mCD99L2- cells (Fig. 5C, lane 4) was weaker compared with that in the A20 and A20-empty cells (Fig. 5C, lanes 1 and 2), which was an evidence of the effect of shRNA targeting mCD99L2. The expression of $\mathrm{p}-\mathrm{I} \kappa \mathrm{B} \alpha$ protein was stronger in the A20-mCD99L2- cells (Fig. 5C, lane 4) compared with that in the A20 and A20-empty cells (Fig. 5C, lanes 1 and 2), suggesting that $N F-\kappa B$ activity was elevated in the A20-mCD99L2- cells.

When the cells were treated with BAY, an inhibitor of the $\mathrm{NF}-\kappa \mathrm{B}$ signaling pathway, $\mathrm{p}-\mathrm{I} \kappa \mathrm{B} \alpha$ protein expression was significantly decreased in the A20-mCD99L2- cells, while mCD99L2 protein expression was not affected (Fig. 5C, lane 3), which suggests a potential correlation between the downregulation of mCD99L 2 and the NF- $\mathrm{B}$ pathway.

\section{Discussion}

Effect of shRNA targeting mCD99L2 on B cell lymphoma. Previous reports have indicated that the overexpression of the full-length CD99 isoform (CD99wt), one of the two distinct proteins produced by the alternative splicing of the CD99 gene transcript, dramatically inhibits cancer cell proliferation, migration and metastasis, whereas the overexpression of the short CD99 isoform (CD99sh) remarkably favors these phenomena (23), while the effects of $m C D 99 L 2$ on tumor cells have not yet been reported. Our previous study confirmed that $m C D 99 L 2$ is expressed in the A20 cell line (18). In the present study, the effective shRNA sequence targeting mCD99L2 was selected and identified, the mCD99L2-downregulated A20 subclones (A20-mCD99L2- cell) were established and the 
Table VII. Upregulated cytokines in excess of 1.5-fold in A20-mCD99L2- cells compared with A20-empty cells.

\begin{tabular}{lrrllllll}
\hline Row & Col & Col & \multicolumn{1}{c}{ Name } & 1 Primary & 2 Primary & 1 Standard & 2 Standard & No. 2/1 \\
\hline 1,2 & 9 & i & CD30T & 7199 & 12042 & 0.0564 & 0.164 & 2.914 \\
1,2 & 10 & j & CD40 & 12714 & 16692 & 0.173 & 0.261 & 1.519 \\
1,2 & 13 & m & CXCL16 & 11067.5 & 14359.5 & 0.137 & 0.212 & 1.548 \\
3,4 & 6 & f & IFN- $\gamma$ & 11977.5 & 15866.5 & 0.156 & 0.243 & 1.5598 \\
3,4 & 13 & m & IL-3 & 8380.5 & 10564 & 0.081 & 0.134 & 1.650 \\
5,6 & 6 & f & IL-12 p40/p70 & 9328.5 & 12161 & 0.101 & 0.1677 & 1.654 \\
7,8 & 6 & f & MIP-1 $\alpha$ & 10985 & 14135.5 & 0.135 & 0.208 & 1.533 \\
\hline
\end{tabular}

mCD99L2, mouse CD99 antigen-like 2. 1, A20-empty cell; 2, A20-mCD99L2 cell.

morphological, biological and phenotypic characteristics of the A20-mCD99L2- cells were investigated in vitro and in vivo for the first time.

Our results strongly suggest that suppressing mCD99L2 may impair the proliferative ability of murine B cell lymphoma. As the A20 cell-induced tumors were pathologically described as DLBCL, which is a type of lymphoma with poor prognosis, our investigation on mCD99L2 may provide a potential target of CD99-related antigens for the clinical therapy of B cell lymphoma.

Downregulation of $m C D 99 L 2$ leads to the transformation of some A20 cells into H/RS-like cells. Using CD99-deficient IM9 and BJAB B cell lines, investigators have confirmed that the downregulation of CD99 is a primary requirement for the generation of H/RS cells $(19,20)$. A previous study of ours suggested that the CD99-upregulated H/RS cell line (L428) lost its nature as an H/RS cell line (21). Although a functional similarity between CD99 and mCD99L2 in lymphoma cells has yet to be confirmed, unique morphological changes were observed in the transformed A20-mCD99L2- cells; some giant cells similar to human H/RS cells were observed in the cultured cells, as well as in nude and BALB/c mice, which suggests that the downregulation of mCD99L2 led to the transformation of some A20 cells into H/RS-like cells.

Biologically, the suppressive effect of the downregulation of $m C D 99 L 2$ on the proliferative ability of mouse B lymphoma cells was observed in the cultured cells and tumor tissues. Although the A20-mCD99L2 - cells grew at a slower rate compared with the A20 cells, cell cycle analysis showed that the $S$ phase of each group did not vary significantly. However, the G2 phase of the A20-mCD99L2- cells lasted for a longer period of time. The fact that some A20-mCD99L2- cells stay in the $\mathrm{G} 2$ phase and exhibit difficulty in entering the $\mathrm{M}$ phase may be due to the fact that giant cells with two or more nuclei were induced in the transformed A20-mCD99L2- cells. The weaker proliferative ability and prolonged $\mathrm{G} 2$ phase imply that A20-mCD99L2 - cells are similar to H/RS cells to a certain extent; thus defects were noted in the cell cycle regulation as one of several anti-apoptotic mechanisms $(24,25)$.

The vast majority of classical Hodgkin's lymphomas are thought to arise from transformed germinal center B cells due to the loss of $\mathrm{B}$ cell characteristics during antigen selection (26). The H/RS cells possess unique morphological and biological features and phenotypic characteristics, thus various phenotypic changes were detected in these cells. The significantly increased CD30 and CD15 expression and moderately decreased CD19 and CD20 expression indicate that the A20-mCD99L2- cells tend to lose part of their B cell characteristics and gain some phenotypic features of H/RS cells, which are characterized by the high expression of CD15 and CD30 (22). Moreover, in the BALB/c mouse tumor tissues of the A20-mCD99L2- group, typical H/RS-like cells were detected, the CD30 antigen was highly expressed and more $\mathrm{CD}^{+} \mathrm{T}$ lymphocytes were observed.

Our results in vitro and in vivo encouraged us to investigate the cytokine expression in various cell groups for the interaction of cytokines/chemokines, which may lead to an environment in which H/RS cells are able to proliferate, escape from apoptosis and survive host antitumor defense (27-29). The results show that several cytokines, such as CD30T, IL-12p40/p70, IL-3, IFN- $\gamma$, CXCL16, MIP-1 $\alpha$ and CD40 were upregulated, a number of which have been associated with Hodgkin's lymphomas or H/RS cells in previous reports $(30,31)$. For instance, compared with other types of lymphoma, the expression of CD30 and CD40 in Hodgkin's lymphoma is highly expressed (32). IL-3 is overexpressed in H/RS cell lines (30). CXCL16 has also been reported to be expressed in some H/RS cell lines (33). Thus, the cytokine expression profile in the A20-mCD99L2 ${ }^{-}$cells exhibited some similarities to that of $\mathrm{H} / \mathrm{RS}$ cells. Combining our in vitro and in vivo observation, A20-mCD99L2- cells partly mimic the characteristics of human $\mathrm{H} / \mathrm{RS}$ cells.

Effect of shRNA targeting mCD99L2 may involve $N F-\kappa B$ pathway. The A20-mCD99L2- cells were observed to have difficulty in forming tumors in mice with normal immune functions. More $\mathrm{CD}^{+}$lymphocytes infiltrated into the A20-mCD99L2 ${ }^{-}$cell-induced tumor tissues compared with the control group in the BALB/c mice, which suggested that certain immune reactions were induced by certain cell groups. Of the upregulated cytokines in the A20-mCD99L2- cells in the cytokine profile, IFN- $\gamma$, IL-12p40/p70 and MIP-1 $\alpha$ have been reported to be involved in regulating immune func- 
tions between tumor cells and lymphocytes (34). CXCL16 has also been reported to be involved in the tumor anti-host reactions (35). Although details are yet to be elucidated, the differentially expressed cytokines may elucidate some of the mechanisms involved in the effect of the downregulation of mCD99L2 in A20 cells and may provide clues for further study.

As regards the pathways, the constitutive NF- $\mathrm{NB}$ activation is a striking feature and the major pathogenetic mechanism in H/RS cells (36-38); therefore, we focused on the NF- $\kappa \mathrm{B}$ pathway. Our finding suggesting that $\mathrm{p}-\mathrm{I} \kappa \mathrm{B} \alpha$, an indicator of the activation of the NF- $\mathrm{B}$ pathway, was enhanced in the A20-mCD99L2- cells, suggested the elevated NF- $\kappa$ B activity by the downregulation of mCD99L2. Treatment with BAY significantly decreased the $\mathrm{p}-\mathrm{I} \kappa \mathrm{B} \alpha$ level in A20-mCD99L2 cells without affecting mCD99L2 protein expression, suggesting a potential regulatory role of mCD99L2 in the $\mathrm{NF}-\kappa \mathrm{B}$ pathway. Although the downregulation of mCD99L2 may trigger various pathways to regulate several cytokines, our results suggested that the downregulation of mCD99L2 in the A20 cells may correlated with the activated $\mathrm{NF}-\kappa \mathrm{B}$ pathway, which may partly contribute to the morphological, biological and phenotypic changes induced by shRNA targeting mCD99L2 in A20 cells. The regulatory mechanisms between $\mathrm{mCD} 99 \mathrm{~L} 2$ and $\mathrm{NF}-\kappa \mathrm{B}$ require extensive investigation in the future.

In conclusion, the stable mCD99L2-downregulated A20 cell line was established and identified. The effect of shRNA targeting mCD99L2 in vitro and in vivo was observed. The downregulation of mCD99L2 led to the transformation of some A20 cells into H/RS-like cells, impaired the proliferative ability of murine $\mathrm{B}$ cell lymphoma, changed the immunophenotypes, led to differentially expressed cytokines and suggested the involvement of the activated $\mathrm{NF}-\kappa \mathrm{B}$ pathway. Our study provides experimental data for additional studies on the mCD99L2 gene and protein in lymphomas.

\section{Acknowledgements}

This study was supported by the National Natural Science Foundation of China (grant nos. 81071941, 81071659 and 81101537).

\section{References}

1. Levy R, Dilley J, Fox RI, et al: A human thymusleukemia antigen defined by hybridoma monoclonal antibodies. Proc Natl Acad Sci USA 76: 6552-6556, 1979.

2. Bernard G, Raimondi V, Alberti I, et al: CD99 (E2) up-regulates alpha4beta1-dependent $\mathrm{T}$ cell adhesion to inflamed vascular endothelium under flow conditions. Eur J Immunol 30: 3061-3065, 2000.

3. Schenkel AR, Mamdouh Z, Chen X, et al: CD99 plays a major role in the migration of monocytes through endothelial junctions. Nature Immunol 3: 143-150, 2002.

4. Tato CM, Joyce-Shaikh B, Banerjee A, et al: The myeloid receptor PILR $\beta$ mediates the balance of inflammatory responses through regulation of IL-27 production. PLoS One 7: e31680, 2012.

5. Park HJ, Byun D, Lee AH, et al: CD99-dependent expansion of myeloid-derived suppressor cells and attenuation of graft-versushost disease. Mol Cells 33: 259-267, 2012.

6. Dworzak MN, Froschl G, Printz D, et al: CD99 expression in T-lineage ALL: implications for flow cytometric detection of minimal residual disease. Leukemia 18: 703-708, 2004
7. Maitra A, Hansel DE, Argani P, et al: Global expression analysis of well-differentiated pancreatic endocrine neoplasms using oligonucleotide microarrays. Clin Cancer Res 9: 5988-5995, 2003.

8. Kang LC and Dunphy CH: Immunoreactivity of MIC2 (CD99) and terminal deoxynucleotidyl transferase in bone marrow clot and core specimens of acute myeloid leukemias and myelodysplastic syndromes. Arch Pathol Lab Med 130: 153-157, 2006.

9. Diwan AH, Skelton HG III, Horenstein MG, et al: Dermatofibrosarcoma protuberans and giant cell fibroblastoma exhibit CD99 positivity. J Cutan Pathol 35: 647-650, 2008.

10. Ramsay AD, Bates AW, Williams S, et al: Variable antigen expression in hepatoblastomas. Appl Immunohistochem Mol Morphol 16: 140-147, 2008.

11. Suh YH, Shin YK, Kook MC, et al: Cloning, genomic organization, alternative transcripts and expression analysis of CD99L2, a novel paralog of human CD99, and identification of evolutionary conserved motifs. Gene 307: 63-76, 2003.

12. Bixel MG, Petri B, Khandoga AG, et al: A CD99-related antigen on endothelial cells mediates neutrophil but not lymphocyte extravasation in vivo. Blood 109: 5327-5336, 2007.

13. Bixel MG, Li H, Petri B, et al: CD99 and CD99L2 act at the same site as, but independently of, PECAM-1 during leukocyte diapedesis. Blood 116: 1172-1184, 2010.

14. Kim KJ, Kanellopoulos-Langevin C, Merwin RM, et al: Establishment and characterization of BALB/c lymphoma lines with B cell properties. J Immunol 122: 549-554, 1979.

15. Passineau MJ, Siegal GP, Everts M, et al: The natural history of a novel, systemic, disseminated model of syngeneic mouse B-cell lymphoma. Leuk Lymphoma 46: 1627-1638, 2005.

16. Warncke M, Buchner M, Thaller G, et al: Control of the specificity of $\mathrm{T}$ cell-mediated anti-idiotype immunity by natural regulatory T cells. Cancer Immunol Immunother 60: 49-60, 2011.

17. Liu F, Zhang G, Zhou XH, et al: Immuno-characterization of mouse model similar to human diffuse large B cell lymphoma. Zhongguo Shi Yan Xue Ye Xue Za Zhi 18: 655-659, 2010 (In Chinese).

18. Shen LJ, Fang WY, Xie SM, et al: Expression and cloning of mCD99L2 gene from mouse B lymphoma cell line A20 and construction of its eukaryotic expression vector. Nan Fang Yi Ke Da Xue Xue Bao 26: 144-149, 2006 (In Chinese).

19. Kim SH, Choi EY, Shin YK, et al: Generation of cells with Hodgkin's and Reed-Sternberg phenotype through downregulation of CD99 (Mic2). Blood 92: 4287-4295, 1998.

20. Kim SH, Shin YK, Lee IS, et al: Viral latent membrane protein 1 (LMP-1)-induced CD99 down-regulation in B cells leads to the generation of cells with Hodgkin's and Reed-Sternberg phenotype. Blood 95: 294-300, 2000.

21. Huang X, Zhou X, Wang Z, et al: CD99 triggers upregulation of miR-9-modulated PRDM1/BLIMP1 in Hodgkin/Reed-Sternberg cells and induces redifferentiation. Int J Cancer 131: E382-E394, 2012.

22. Burgess SC, Young JR, Baaten BJ, et al: Marek's disease is a natural model for lymphomas overexpressing Hodgkin's disease antigen (CD30). Proc Natl Acad Sci USA 101: 13879-13884, 2004.

23. Scotlandi K, Zuntini M, Manara MC, et al: CD99 isoforms dictate opposite functions in tumour malignancy and metastases by activating or repressing c-Src kinase activity. Oncogene 26: 6604-6618, 2007.

24. Garcia JF, Camacho FI, Morente M, et al: Hodgkin and ReedSternberg cells harbor alterations in the major tumor suppressor pathways and cell-cycle checkpoints: analyses using tissue microarrays. Blood 101: 681-689, 2003.

25. Sánchez-Águilera A, Montalbán C, de la Cueva P, et al: Tumor microenvironment and mitotic checkpoint are key factors in the outcome of classic Hodgkin lymphoma. Blood 108: 662-668, 2006.

26. Marafioti T, Hummel M, Foss HD, et al: Hodgkin and ReedSternberg cells represent an expansion of a single clone originating from a germinal center B-cell with functional immunoglobulin gene rearrangements but defective immunoglobulin transcription. Blood 95: 1443-1450, 2000.

27. Maggio E, van den Berg A, Diepstra A, et al: Chemokines, cytokines and their receptors in Hodgkin's lymphoma cell lines and tissues. Ann Oncol 13 (Suppl 1): 52-56, 2002.

28. Skinnider BF and Mak TW: The role of cytokines in classical Hodgkin lymphoma. Blood 99: 4283-4297, 2002.

29. Steidl C, Connors JM and Gascoyne RD: Molecular pathogenesis of Hodgkin's lymphoma: increasing evidence of the importance of the microenvironment. J Clin Oncol 29: 1812-1826, 2011. 
30. Aldinucci D, Olivo K, Lorenzon D, et al: The role of interleukin-3 in classical Hodgkin's disease. Leuk Lymphoma 46: 303-311, 2005.

31. Niederkorn JY: Emerging concepts in CD8(+) T regulatory cells Curr Opin Immunol 20: 327-331, 2008.

32. Lee IS, Kim SH, Song HG, et al: The molecular basis for the generation of Hodgkin and Reed-Sternberg cells in Hodgkin's lymphoma. Int J Hematol 77: 330-335, 2003.

33. Hanamoto $\mathrm{H}$, Nakayama $\mathrm{T}$, Miyazato $\mathrm{H}$, et al: Expression of CCL28 by Reed-Sternberg cells defines a major subtype of classical Hodgkin's disease with frequent infiltration of eosinophils and/or plasma cells. Am J Pathol 164: 997-1006, 2004.

34. Luft T, Luetjens P, Hochrein H, et al: IFN-alpha enhances CD40 ligand-mediated activation of immature monocyte-derived dendritic cells. Int Immunol 14: 367-380, 2002.
35. Meijer J, Ogink J, Kreike B, et al: The chemokine receptor CXCR6 and its ligand CXCL16 are expressed in carcinomas and inhibit proliferation. Cancer Res 68: 4701-4708, 2008.

36. Horie R, Watanabe T, Morishita Y, et al: Ligand-independent signaling by overexpressed CD30 drives NF-kappaB activation in Hodgkin-Reed-Sternberg cells. Oncogene 21: 2493-2503, 2002.

37. Aldinucci D, Lorenzon D, Cattaruzza L, Pinto A, Gloghini A Carbone A and Colombatti A: Expression of CCR5 receptors on Reed-Sternberg cells and Hodgkin lymphoma cell lines: involvement of CCL5/Rantes in tumor cell growth and microenvironmental interactions. Int J Cancer 122: 769-776, 2008.

38. Schwarzer R and Jundt F: Notch and NF- $\mathrm{KB}$ signaling pathways in the biology of classical Hodgkin lymphoma. Curr Mol Med 11: 236-245, 2011. 